\title{
A Loss Reduction Optimization Method for Distribution Network Based on Combined Power Loss Reduction Strategy
}

\author{
Jihua Xie, Chang Chen, and Huan Long (iD \\ School of Electrical Engineering, Southeast University, Nanjing 210098, China \\ Correspondence should be addressed to Huan Long; hlong@seu.edu.cn
}

Received 11 June 2021; Accepted 13 July 2021; Published 23 July 2021

Academic Editor: Long Wang

Copyright (c) 2021 Jihua Xie et al. This is an open access article distributed under the Creative Commons Attribution License, which permits unrestricted use, distribution, and reproduction in any medium, provided the original work is properly cited.

Power loss reflects the effective utilization rate of energy and the management level of power grids. In this paper, we propose a combined power loss reduction strategy optimization framework to improve the power loss reduction effect in a distribution network. The weak points of the distribution network are analyzed based on power flow calculation. The corresponding power loss reduction strategies are generated considering the following three aspects: replacing distribution lines, distribution transformers, and reactive power compensation. A combined power loss reduction strategy optimization model considering the comprehensive benefits of power loss reduction is established. A method for solving the optimization model based on the cost-benefit ratio is also proposed. Experiments based on the dataset from Tianjin show that the proposed loss reduction optimization method can effectively reduce power loss and formulate a reasonable loss reduction modification scheme in the distribution network.

\section{Introduction}

Power loss rate is an essential comprehensive index to measure the technical management and operation management levels of power supply enterprises. Since the power loss of the distribution network occupies a considerable proportion in the whole power system, the loss reduction modification of the distribution network has always been the critical work for power supply enterprises to improve their economic operation [1-4]. Thus, loss reduction optimization for the distribution network is a vital problem for power supply enterprises.

Loss reduction strategies of a distribution network can be mainly divided into management and technical strategies. Since the management strategies are primarily related to human factors, the primary task of power supply enterprises is to optimize the power loss management system and standardize the power loss management process [5-8]. Thus, the technical strategies of loss reduction are mainly taken into consideration in this paper.

The current research work on the loss reduction of the distribution network has been studied from many aspects. In [9], various loss reduction technical strategies of the distribution network were comprehensively summarized from two aspects of power equipment configuration and grid system operation. In [10], an evolutionary programmingbased technique was proposed to optimize the placement of distributed generation units energized by wind and solar energy in a radial distribution system. In [11], based on considering the stochastic nature of distributed generation, a comprehensive optimization model for the simultaneous allocation of capacitor banks and distributed generation was proposed, and a hybrid algorithm based on Tabu search and genetic algorithms was also proposed to solve the model. In [12], on the basis of considering the uncertainty of distributed generation, electric vehicles, and other loads, Latin hypercube sampling was employed to generate random variables, and a bilayer optimization model was constructed. The improved harmony search algorithm was used to realize the dynamic reconfiguration of the distribution network. In [13], the multiobjective distribution network reconfiguration model considering distributed power generation and load uncertainty was proposed, which could optimize multiple important goals of the distribution network and effectively reduce the power loss of the distribution network. In [14], combining a microscopic analysis and the macro 
statistics of the distribution network, an energy saving modification investment planning model, constrained by the investment and weighting factors, was developed to evaluate the energy saving. In [15], the Bat algorithm was used to solve the problem of reactive power source optimization for bus voltage deviation index minimization by the optimal placement of a number of capacitor banks in the network buses. In [16], optimal D-STATCOM placement and size was determined based on the index vector method for radial distribution networks under a reconfigured network to reduce the power loss. In [17], a multiobjective evolutionary algorithm based on a fuzzy decision-making method was proposed to reduce the power loss and improve the reliability of the radial distribution system.

Although there are currently a large number of references on loss reduction strategies, they mainly focus on the theoretical elaboration of different loss reduction strategies. Previous studies are mostly focused on calculating the power saving amount of various specific strategies such as reactive power compensation and power equipment replacement and analyzing the effect of energy saving and loss reduction of different strategies [18]. However, there is little research on the selection method of loss reduction modification scheme based on the combination of multiple loss reduction strategies, leading to the possibility that the loss reduction effect may not be the optimal situation.

In order to solve the abovementioned problems, a novel loss reduction optimization method for the distribution network is proposed in this paper based on the combined power loss reduction strategy that is divided into three stages: weak point analysis of power loss, generation of loss reduction strategy, and combined loss reduction strategy optimization. The weak point analysis of power loss of the distribution network is first carried out based on power flow calculation. The corresponding power loss reduction strategies are then generated considering three aspects: replacing distribution lines, distribution transformers, and reactive power compensation. A combined power loss reduction strategy optimization model considering the comprehensive benefits of power loss reduction is established. A method for solving the optimization model based on the cost-benefit ratio is proposed. The dataset from a power supply company in Tianjin is utilized to validate the proposed methodology.

\section{Methodology}

2.1. The Structure of the Proposed Algorithm. Although there are many loss reduction technical strategies in the current distribution network, there is little research on loss reduction optimization based on a combination of multiple types of loss reduction strategies. The current loss reduction strategies are relatively simple and lack pertinence. Thus, a framework of combined loss reduction strategy optimization in the distribution network is proposed in this paper, as shown in Figure 1, which is mainly divided into three stages: weak point analysis of power loss, generation of loss reduction strategy, and combined loss reduction strategy optimization.
In the stage of weak point analysis of power loss, considering that the load of distribution transformers is constantly changing during the operation of the distribution network, in order to make the results of loss reduction analysis more consistent with the actual situation, the clustering algorithm is employed to generate typical load curves for all distribution transformers to establish a typical loss reduction scenario. Based on the statistical analysis of the loss operation data through the power flow calculation, the weak points of the power loss of the distribution network feeder can be identified, including the severely aged branches, the distribution transformers with low power factor, and the branches with excessive power loss. In the stage of generation of loss reduction strategy, based on the results of the weak point analysis, the corresponding loss reduction strategies are generated for each loss reduction object (distribution transformer, distribution line, etc.), considering the three aspects of replacing distribution lines, distribution transformers, and reactive power compensation. The commonly used energy saving and loss reduction strategies are depicted in Figure 2. Finally, in the stage of combined loss reduction strategy optimization, to take into account the loss reduction effect and economy, a combined loss reduction strategy optimization model for the distribution network is established. A novel method for solving the above optimization model based on the cost-benefit ratio is proposed to optimize the solution process and formulate a reasonable combined strategy for loss reduction modification scheme for the distribution network.

\subsection{Combined Loss Reduction Strategy Optimization Model.} The loss reduction modification scheme of the distribution network is composed of different types of loss reduction strategies. Each type of loss reduction strategy has a variety of specific implementation situations for choice. When formulating a loss reduction modification scheme, it is necessary to consider the loss reduction effect of the distribution network feeder after the loss reduction modification and to analyze the economy of the loss reduction modification.

2.2.1. Objective Function. This paper mainly generates loss reduction strategies from distribution transformer, distribution line, and reactive power compensation of distribution network. Thus, the cost of power loss, the replacement cost of distribution lines, the replacement cost of distribution transformers, and the cost of reactive power compensation are needed to be considered. To optimize the comprehensive benefits of loss reduction, the objective function of the combined loss reduction strategy optimization model is established as shown in the following equation:

$$
\min C=C_{\text {loss }}^{i}+C_{\mathrm{vc}}^{i}+C_{l}^{i}+C_{t}^{i},
$$

where $C$ represents the sum of costs involved in the loss reduction modification scheme with multiple strategies combination; $C_{\text {loss }}^{i}$ represents the power loss cost of the $i$-th loss reduction modification scheme; $C_{\mathrm{vc}}^{i}$ denotes the reactive 


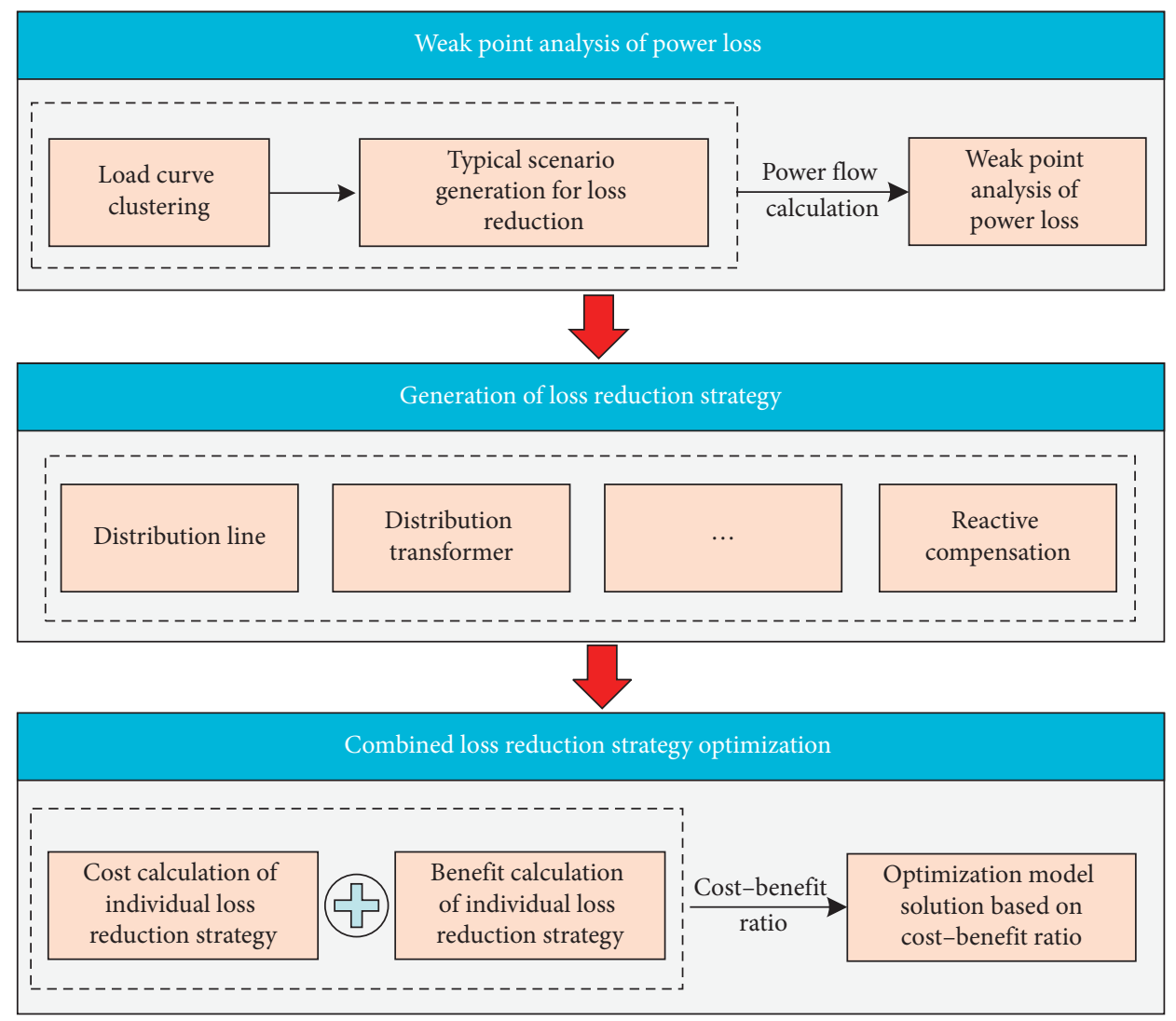

FIgURE 1: The proposed framework of combined loss reduction strategy optimization.

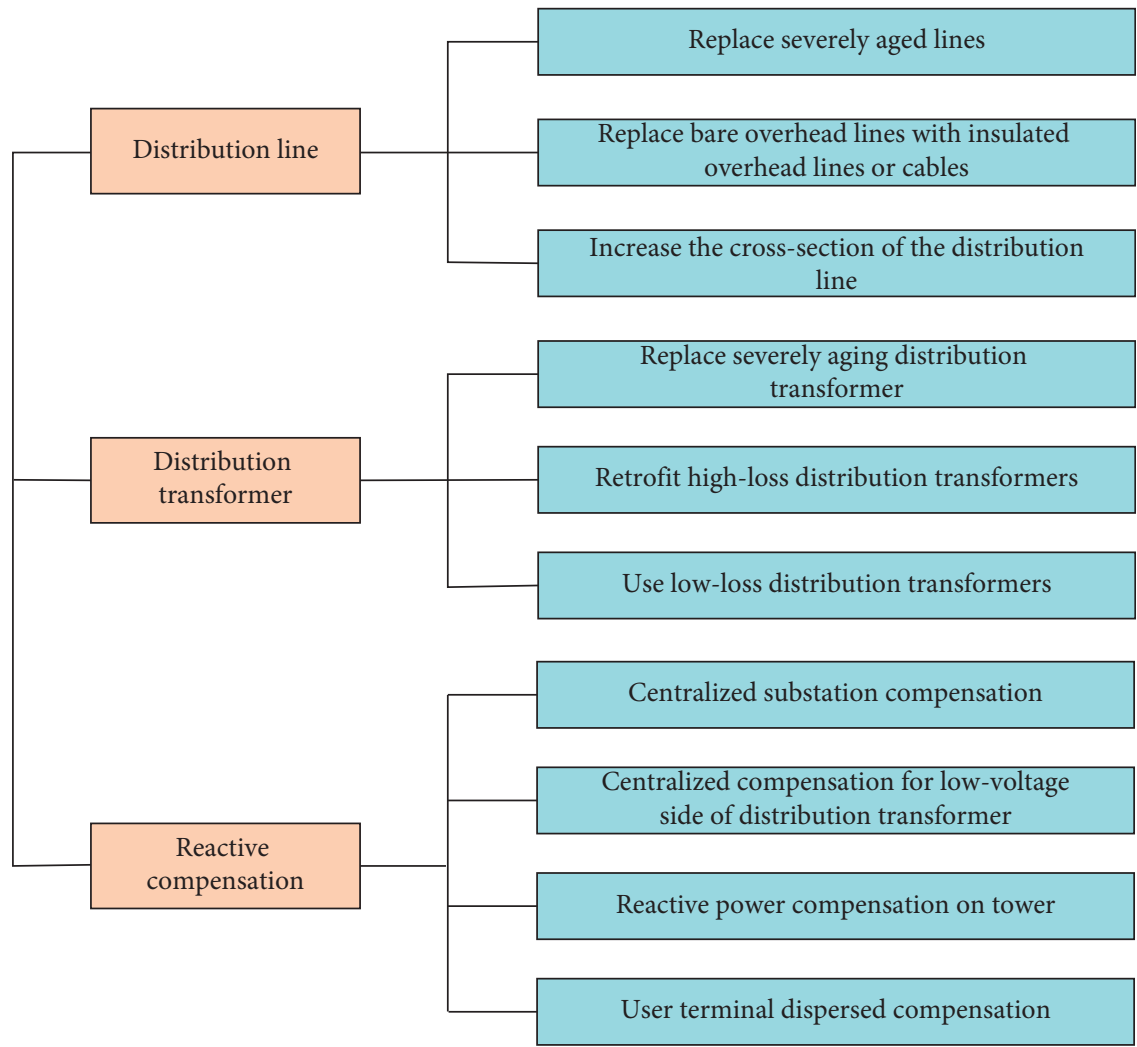

FIgURE 2: Commonly used energy saving and loss reduction strategies of the distribution network. 
power compensation cost of the $i$-th loss reduction modification scheme; $C_{l}^{i}$ stands for the replacement cost of the distribution line of the $i$-th loss reduction modification scheme; and $C_{t}^{i}$ denotes the replacement cost of the distribution transformer of the $i$-th loss reduction modification scheme.

(1) Power Loss Cost. Power loss cost refers to the loss of electricity sale cost caused by power loss, which can be calculated by multiplying the power loss quantity with the corresponding electricity price, as shown in the following equation:

$$
C_{\mathrm{loss}}^{i}=\sum_{j=1}^{24} P_{\mathrm{loss}}^{i, j} \cdot \tau_{e} \cdot n_{\mathrm{LT}},
$$

where $P_{\text {loss }}^{i, j}$ represents the power loss at the $j$-th hour of the $i$-th loss reduction modification scheme; $\tau_{e}$ represents the electricity price; and $n_{\mathrm{LT}}$ represents the time frame considered for power loss cost, set as 365 in this paper.

(2) Reactive Power Compensation Cost. In this paper, the total compensated reactive power capacity of the distribution network is used to estimate its reactive power compensation cost, as shown in the following equation:

$$
C_{\mathrm{vc}}^{i}=\sum_{j=1}^{24} \sum_{k_{\mathrm{vc}}=1}^{N_{\mathrm{vc}}^{i}} Q_{k_{\mathrm{vc}}}^{i, j} \cdot \tau_{\mathrm{vc}} \cdot n_{\mathrm{LT}},
$$

where $N_{\mathrm{vc}}^{i}$ represents the number of reactive power compensation points of the $i$-th loss reduction modification scheme; $Q_{k_{\mathrm{vc}}}^{i, j}$ denotes the reactive power compensation capacity at the $j$-th hour at the $k$-th point of the $i$-th loss reduction modification scheme; and $\tau_{\mathrm{vc}}$ represents the unit construction cost for reactive power compensation.

(3) Replacement Cost of the Distribution Line. The replacement cost of the distribution line is related to the length and type of the line, which is calculated using the following equation:

$$
C_{l}^{i}=\sum_{k_{l}=1}^{N_{l}^{i}} L_{k_{l}}^{i} \cdot \tau_{l, k_{l}}^{i}
$$

where $N_{l}^{i}$ represents the number of distribution lines that need to be replaced in the $i$-th loss reduction modification scheme; $L_{k_{l}}^{i}$ represents the length of the $k_{l}$-th distribution line to be replaced in the $i$-th loss reduction modification scheme; and $\tau_{l, k l}^{i}$ represents the unit construction cost of the $k_{l}$-th distribution line to be replaced in the $i$-th loss reduction modification scheme.

(4) Replacement Cost of the Distribution Transformer. The replacement cost of the distribution transformer is related to the type and capacity of the distribution transformer, which is described in the following equation:

$$
C_{t}^{i}=\sum_{k_{t}=1}^{N_{t}^{i}} \tau_{t, k_{t}}^{i}
$$

where $N_{t}^{i}$ represents the number of distribution transformers that need to be replaced in the $i$-th loss reduction modification scheme and $\tau_{t, k t}^{i}$ represents the unit construction cost of the $k_{t}$-th distribution transformer to be replaced in the $i$-th loss reduction modification scheme.

\subsubsection{Constraints}

(1) Power Loss Rate Constraint. Based on the development goals of the electric power development plan, power supply enterprises usually set the target value of the power loss rate after loss reduction modification, expressed in equation (6). For example, in the "13th Five-Year Plan for Electric Power Development (2016-2020)," the target value of the power loss rate is $6.5 \%$.

$$
P_{\text {loss }} \%=\frac{P_{\text {sup }}-P_{\text {sales }}}{P_{\text {sup }}} \times 100 \%<\eta,
$$

where $P_{\text {loss }} \%$ represents the power loss rate of the distribution network feeder after loss reduction modification; $P_{\text {sup }}$ represents the power supply; $P_{\text {sales }}$ represents the power sale quantity; and $\eta$ represents the target value of the power loss rate after loss reduction modification of the distribution network feeder.

\section{(2) Power Flow Constraint}

$$
\left\{\begin{array}{l}
P_{i}=U_{i} \sum_{j \in i} U_{j}\left(G_{i j} \cos \delta_{i j}+B_{i j} \sin \delta_{i j}\right), \\
Q_{i}=U_{i} \sum_{j \in i} U_{j}\left(G_{i j} \sin \delta_{i j}-B_{i j} \cos \delta_{i j}\right),
\end{array}\right.
$$

where $P_{i}$ represents the active power injected into the bus $i$; $Q_{i}$ represents the reactive power injected to the bus $i ; U_{i}$ represents the voltage of bus $i ; \delta_{i j}$ denotes the phasor between bus $i$ and $j$; $G_{i j}$ denotes the conductance between bus $i$ and $j ; B_{i j}$ represents the susceptance between bus $i$ and $j$; and $G_{i i}+j B_{i i}$ represents the self-admittance of bus $i$.

(3) Branch Transmission Capacity Constraint. The actual transmission capacity of the branch usually cannot exceed the maximum transmission capacity of the branch. In order to make the current operate within the normal range, the branch transmission capacity constraint is expressed in the following equation:

$$
0 \leq I_{i j} \leq I_{\max }
$$

where $I_{\max }$ is the maximum allowable flow carrying capacity of the branch.

(4) Node Voltage Constraint. In order to make the node voltage operate within the normal range, the node voltage constraint is expressed as shown in the following equation: 


$$
U_{\min } \leq U_{i} \leq U_{\max }
$$

where $U_{\min }$ and $U_{\max }$ are the minimum and maximum values of the node voltage, respectively.

(5) Reactive Power Compensation Capacity Constraint. The constraint of reactive power compensation capacity is shown in the following equation:

$$
Q_{i, \min } \leq Q_{i} \leq Q_{i, \max },
$$

where $Q_{i}$, min and $Q_{i}, \max$ represent the minimum and maximum values of reactive power compensation capacity at bus $i$, respectively.

2.2.3. Solution Method Based on Cost-Benefit Ratio. The purpose of solving the combined loss reduction strategy optimization model is to optimize the set of multiple loss reduction modification schemes composed of different loss reduction strategies for all loss reduction objects (distribution lines, distribution transformers, etc.), formulating a loss reduction modification scheme with the best comprehensive benefit of loss reduction, considering both the effect of loss reduction and the economy of loss reduction. The current research generally solves the combined loss reduction strategy optimization model through the enumeration method. For the alternative loss reduction modification schemes that meet the constraints, the objective function values are directly compared to determine the final loss reduction modification scheme with the best comprehensive benefit of loss reduction.

However, the number of alternative loss reduction modification schemes is closely related to the number of loss reduction objects determined by power loss weak point analysis results and the number of corresponding loss reduction strategies. Thus, there may be a huge number of alternative loss reduction modification schemes, which will inevitably lead to a large amount of calculation, resulting in low solution efficiency of the optimization model. A solution method based on the cost-benefit ratio is proposed in this paper to solve the above problem.

In this paper, the cost-benefit ratio, $\mu_{\mathrm{LR}}$, represents the ratio of the cost of loss reduction, $C_{\mathrm{LR}}$, to the benefit of loss reduction, $B_{L R}$, as shown in equation (11). $C_{\mathrm{LR}}$ consists of the replacement cost of distribution lines, the replacement cost of distribution transformers, and the cost of reactive power compensation, described in equation (12). $B_{\mathrm{LR}}$ is the cost corresponding to the loss reduction electricity after the loss reduction modification in equation (13). It can be seen that when $C_{\mathrm{LR}}$ is lower and $B_{\mathrm{LR}}$ is higher, the corresponding $\mu_{\mathrm{LR}}$ is smaller, which means that the corresponding loss reduction strategy should be selected.

$$
\begin{aligned}
& \mu_{\mathrm{LR}}=\frac{C_{\mathrm{LR}}}{B_{\mathrm{LR}}}, \\
& C_{\mathrm{LR}}=C_{\mathrm{vc}}+C_{l}+C_{t},
\end{aligned}
$$

$$
B_{\mathrm{LR}}=C_{\mathrm{loss} 1}-C_{\mathrm{loss} 2},
$$

where $C_{\text {loss } 1}$ represents the power loss cost of the distribution network feeder before the loss reduction modification and $C_{\text {loss2 }}$ denotes the power loss cost after the loss reduction modification.

The solution process of the combined loss reduction strategy optimization model based on the cost-benefit ratio is shown in Figure 3. The specific steps are described as follows:

Step 1: based on the loss reduction strategies generated by the results of power loss weak point analysis, the loss reduction strategies that meet the constraints (power flow constraint, branch transmission capacity constraint, node voltage constraint, and reactive power compensation capacity constraint) are selected through power flow calculation. Then, the loss reduction cost, loss reduction benefit, and cost-benefit ratio when each loss reduction strategy is implemented separately are calculated.

Step 2: according to the order of cost-benefit ratio, the loss reduction strategies with the lowest cost-benefit ratio of each loss reduction object are selected and determined as the individual optimal loss reduction strategy corresponding to the loss reduction object.

Step 3: based on the individual optimal loss reduction strategies determined in Step 2, a set of alternative strategies for the loss reduction modification scheme are constructed according to the order of the costbenefit ratio from low to high.

Step 4: the number of loss reduction strategies in the loss reduction modification scheme, $r$, is set to 1 .

Step 5: the first $r$ alternative loss reduction strategies are combined to construct a loss reduction modification scheme, and a loss reduction cost-benefit analysis is conducted based on the power flow calculation.

Step 6: if the termination condition is not met, then $r=r+1$, continue to Step 5; if the termination condition is met, the current loss reduction modification scheme is determined as the final distribution network loss reduction modification scheme. The termination condition in this paper is that $P_{\text {loss }} \%<\eta$.

It is worth noting that if the loss reduction modification scheme combines all the alternative strategies and cannot be less than the target power loss rate, it is necessary to regenerate loss reduction strategies with better loss reduction effects based on the power loss weak points.

\section{Case Study}

In this section, the data used in the experiment are first described. The loss reduction result of the selected feeder of the distribution network is displayed in the remaining sections. 


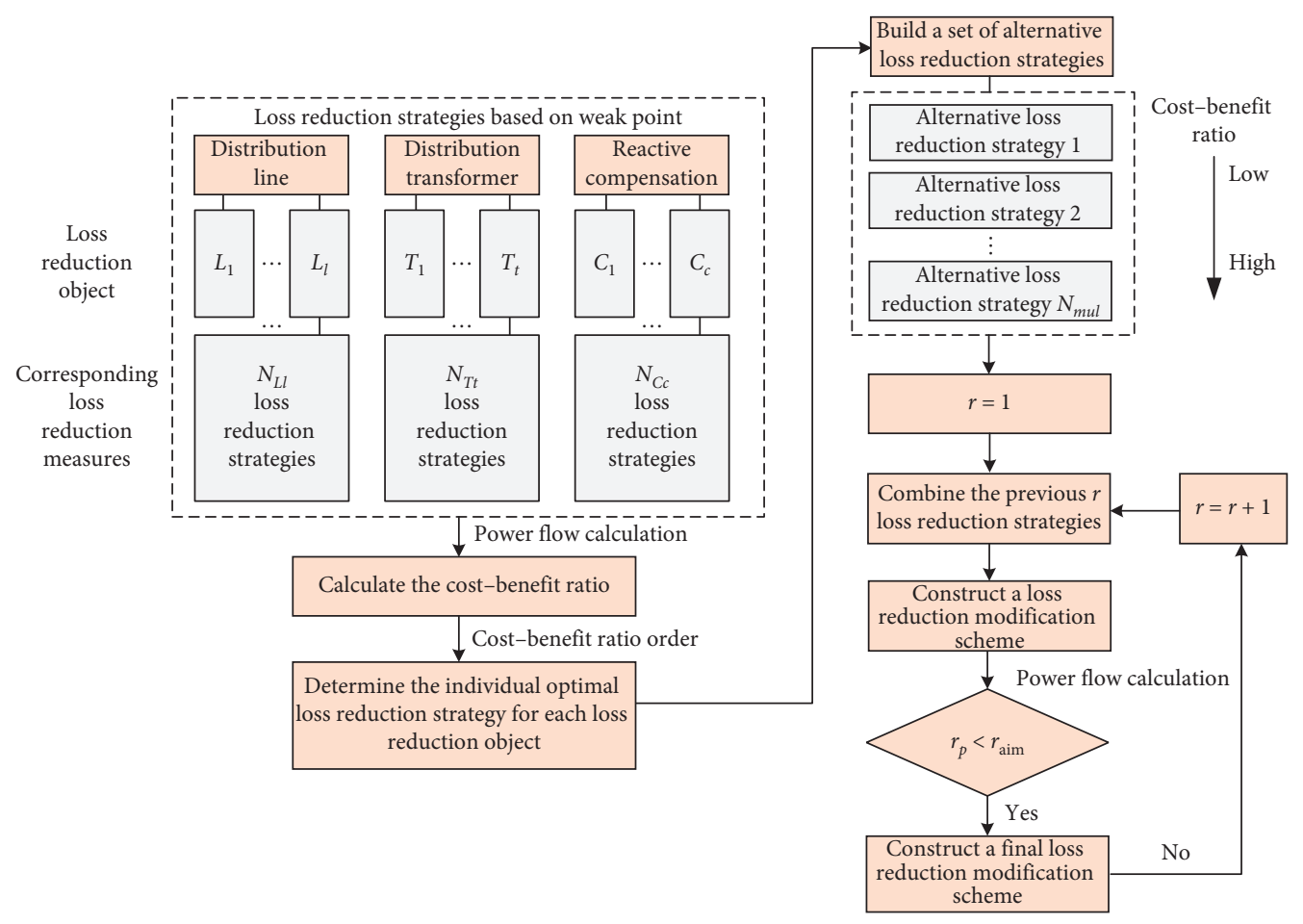

FIGURE 3: The solution process of the combined loss reduction strategy optimization model based on the cost-benefit ratio.

3.1. Dataset. The dataset utilized in this paper was collected from the Tianjin Electric Power Company in China. A $10 \mathrm{kV}$ feeder of the distribution network is selected to conduct loss reduction, the topology of which is shown in Figure 4 . The data, covering from January 1, 2019, to December 31, 2019, include the topology parameters, the parameters of power equipment, and load.

Compared with normal feeders, the feeders in the distribution network that need loss reduction generally have a higher power loss rate with a part of aged power equipment. Thus, in order to simulate the aging situation, the relevant parameters of the distribution lines, distribution transformers, and loads are modified to a certain extent. The specific modification is shown in Table 1 . The parameter modification method of the aged transformer and the aged lines is to change their resistance parameters. In this paper, their resistance parameters are increased to $1.04 \sim 1.14$ times of the original values [19].

3.2. Typical Scenario Generation for Loss Reduction. In order to generate a typical loss reduction scenario, the daily load curves sampled every 15 minutes of each distribution transformer in Figure 4 are clustered based on K-means [20,21]. Taking transformer $T 1$ as an example, the load clustering results of transformer is shown in Figure 5. Figure 6 shows the center curves of the three clusters with the largest number of samples of part of distribution transformers. The cluster center of the cluster with the largest number of samples for each distribution transformer is taken as the typical load curve of each distribution transformer in a typical loss reduction scenario.
3.3. Generation of Loss Reduction Strategy. Based on the typical loss reduction scenario, the result of the power flow calculation is that the power loss rate of the feeder in Figure 4 is $6.4 \%$, and its average power factor is 0.9 when no loss reduction strategies are selected in this typical scenario. The power factor of each public transformer is shown in Figure 7.

In order to generate targeted loss reduction strategies, according to the power flow calculation results and the power equipment parameters set above, the loss reduction strategies for each loss reduction object are proposed from the three aspects of distribution lines, transformers, and reactive power compensation. The details of the specific loss reduction strategies presented in this section are shown in Tables 2-4, the specific new type numbers of which are shown in Tables S1-S3 in Supplementary Material, respectively. The main ideas for generating loss reduction strategies in this paper are as follows:

(1) Distribution line: for severely aged lines or bare overhead conductors, replace them with new conductors or build new overhead insulated conductors/ cables with the same cross-section according to actual conditions. The conductors can also be expanded accordingly considering the development requirements of the load;

(2) Distribution transformers: replace S7 series and other high-loss old transformers with S11, S13, or amorphous alloy-type distribution transformers;

(3) Reactive power compensation: select the distribution transformer with a power factor less than 0.85 to select reactive power compensation strategies, 


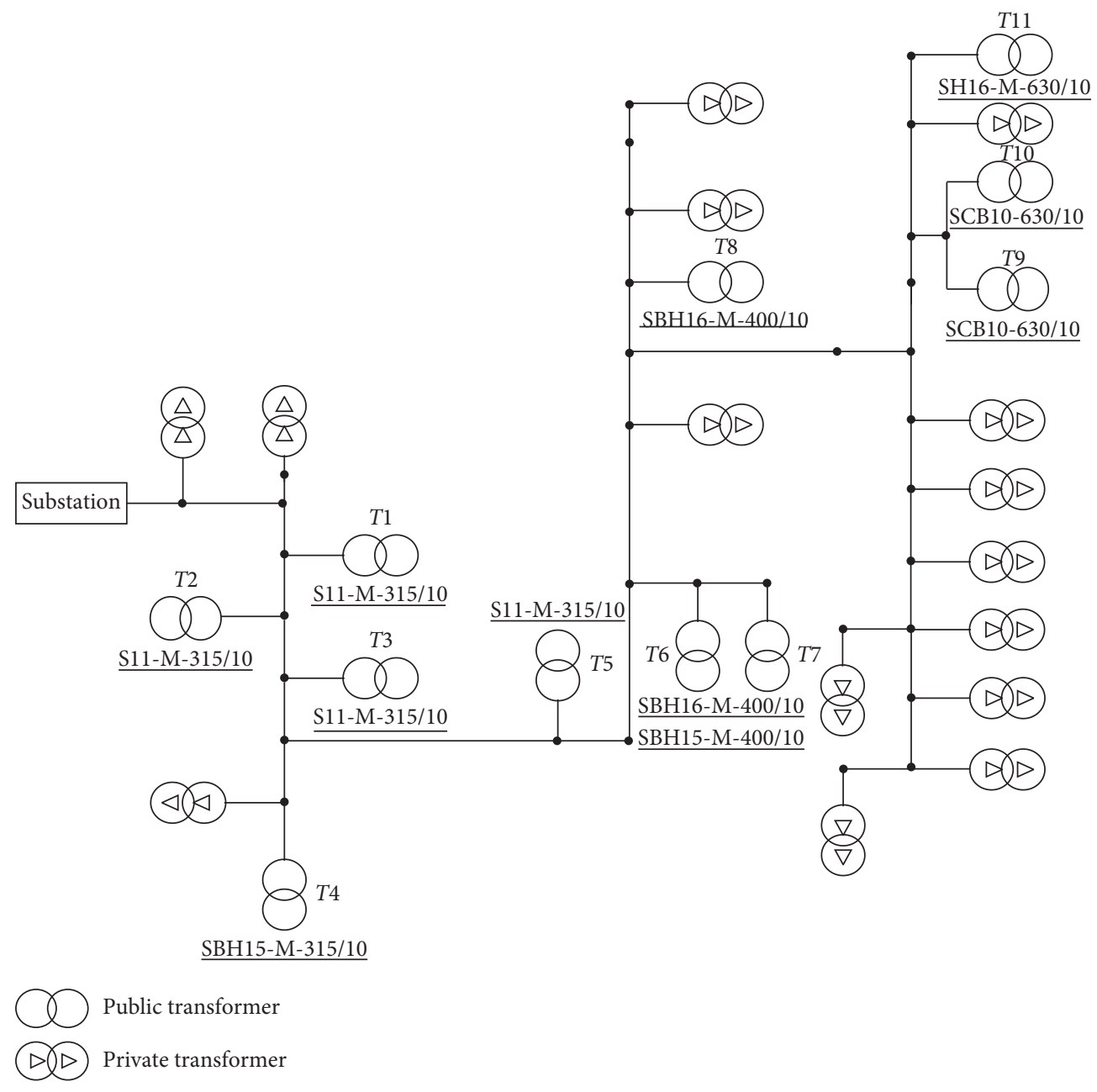

FIgURE 4: The selected feeder topology for loss reduction.

TAвLE 1: Parameter modification details of transformers and lines.

\begin{tabular}{|c|c|c|c|}
\hline Equipment & Head node of branch & Tail node of branch & Parameter modification details \\
\hline \multirow{7}{*}{ Transformer } & 13 & 14 & \multirow{2}{*}{ Change the equipment type to type 11} \\
\hline & 16 & 17 & \\
\hline & 18 & 19 & \multirow{3}{*}{ Change the equipment type to type 11 and set a certain degree of aging } \\
\hline & 27 & 28 & \\
\hline & 51 & 52 & \\
\hline & 51 & 53 & \multirow{2}{*}{ Change the equipment type to type 7} \\
\hline & 55 & 56 & \\
\hline \multirow{8}{*}{ Line } & 12 & 13 & \multirow{8}{*}{ Set a certain degree of aging } \\
\hline & 18 & 20 & \\
\hline & 21 & 22 & \\
\hline & 22 & 23 & \\
\hline & 26 & 27 & \\
\hline & 34 & 35 & \\
\hline & 35 & 37 & \\
\hline & 41 & 42 & \\
\hline
\end{tabular}




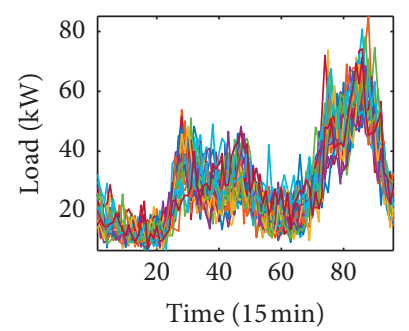

(a)

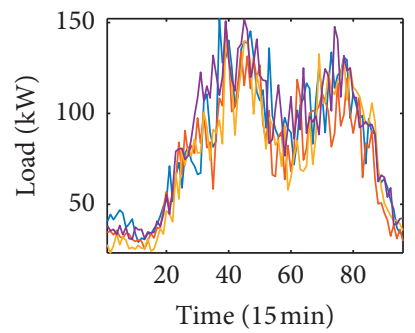

(e)

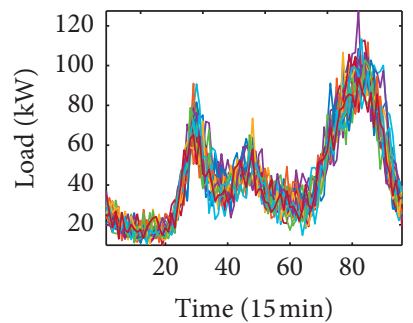

(i)

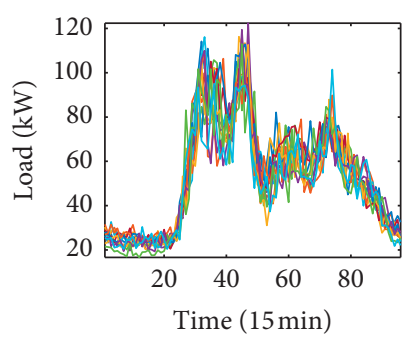

(b)

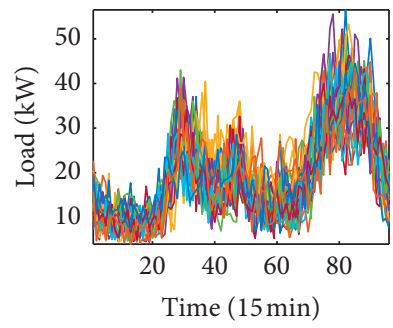

(f)

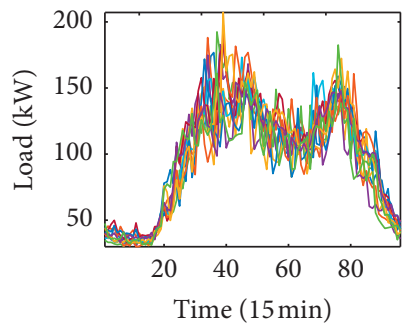

(j)

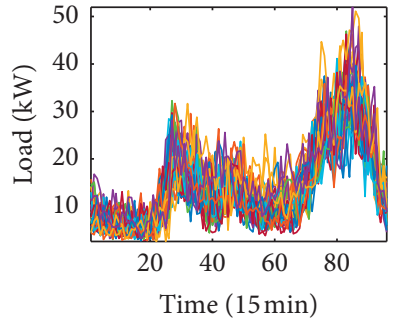

(c)

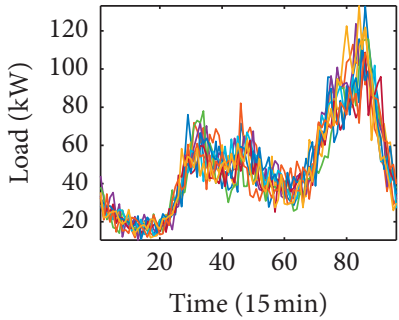

(g)

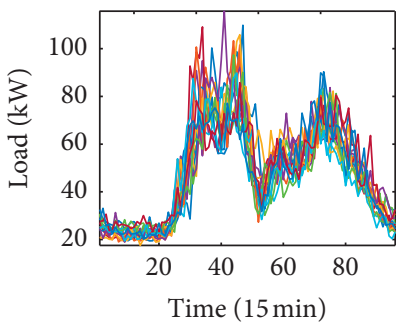

(k)

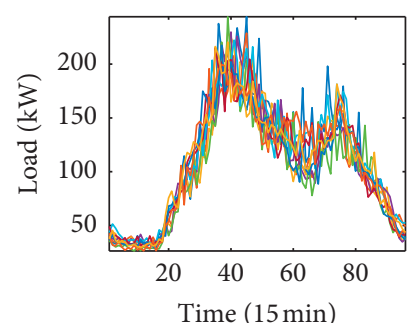

(d)

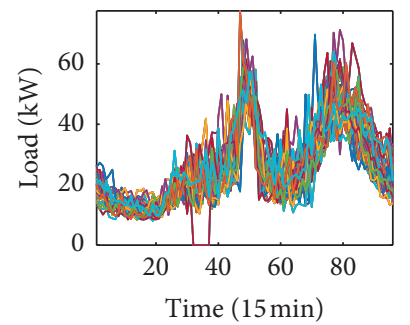

(h)

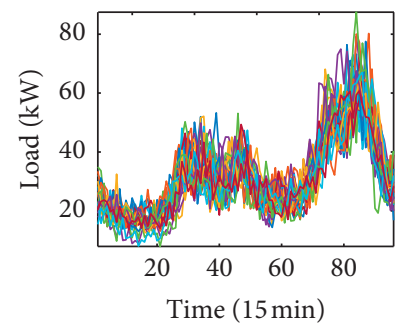

(1)

FIgURE 5: Load clustering results of transformer $T 1$.

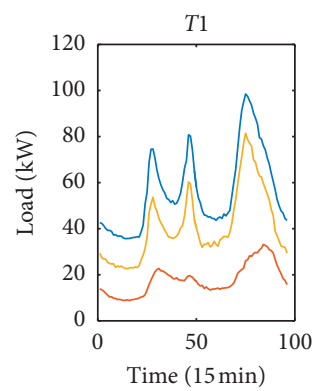

(a)

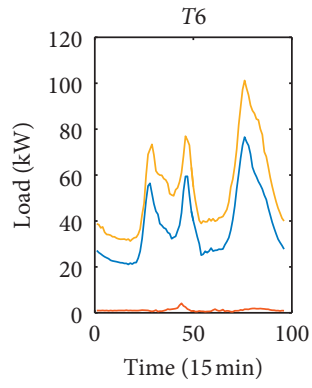

(f)

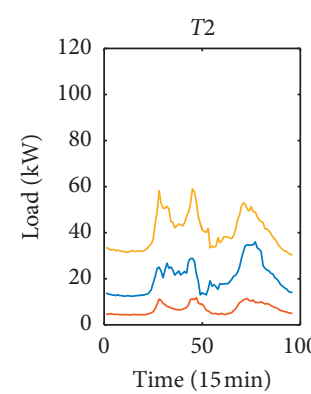

(b)

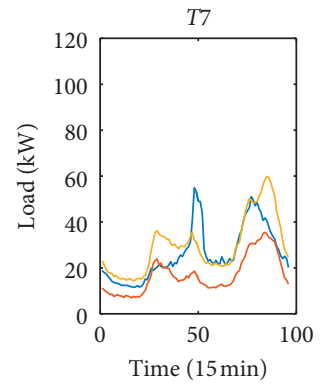

(g)

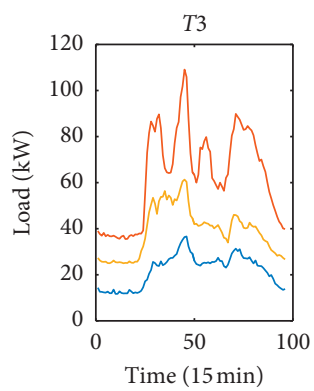

(c)

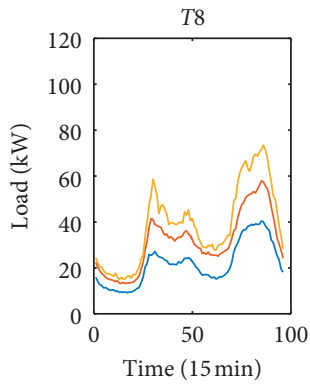

(h)

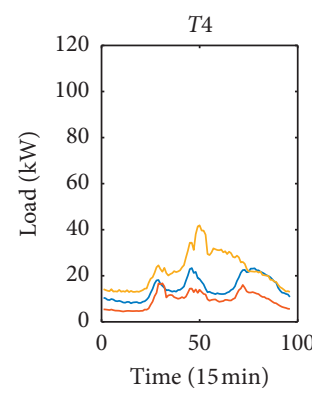

(d)

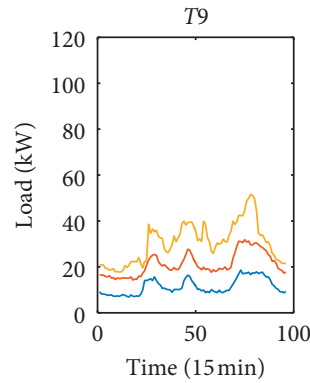

(i)

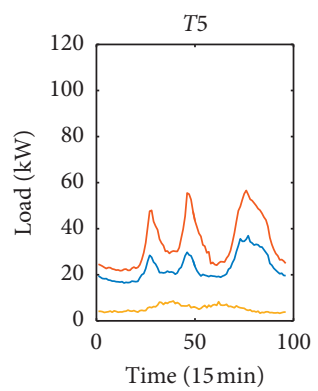

(e)

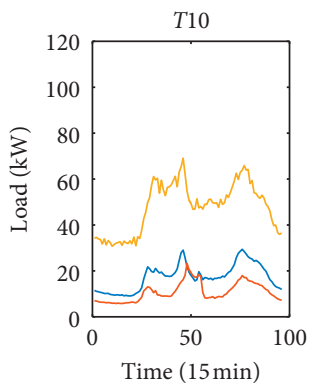

(j)

Figure 6: The center curves of the three clusters with the largest number of samples of part of distribution transformers. 


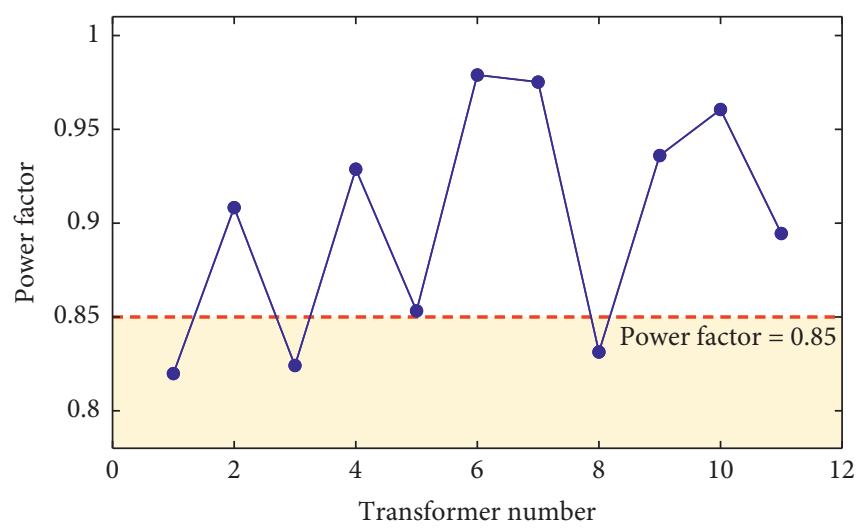

Figure 7: The power factor of each public transformer.

TABLe 2: Individual loss reduction strategies (distribution line).

\begin{tabular}{|c|c|c|c|c|c|c|c|}
\hline $\begin{array}{l}\text { Strategy } \\
\text { number }\end{array}$ & $\begin{array}{l}\text { Head node of } \\
\text { branch }\end{array}$ & $\begin{array}{c}\text { Tail node of } \\
\text { branch }\end{array}$ & $\begin{array}{c}\text { New type } \\
\text { number }\end{array}$ & $\begin{array}{l}\text { Strategy } \\
\text { number }\end{array}$ & $\begin{array}{l}\text { Head node of } \\
\text { branch }\end{array}$ & $\begin{array}{c}\text { Tail node of } \\
\text { branch }\end{array}$ & $\begin{array}{c}\text { New type } \\
\text { number }\end{array}$ \\
\hline 1 & 12 & 13 & 9 & 18 & 34 & 35 & 11 \\
\hline 2 & 12 & 13 & 10 & 19 & 34 & 35 & 17 \\
\hline 3 & 12 & 13 & 17 & 20 & 35 & 37 & 10 \\
\hline 4 & 12 & 13 & 6 & 21 & 35 & 37 & 11 \\
\hline 5 & 18 & 20 & 10 & 22 & 35 & 37 & 17 \\
\hline 6 & 18 & 20 & 11 & 23 & 41 & 42 & 10 \\
\hline 7 & 18 & 20 & 17 & 24 & 41 & 42 & 11 \\
\hline 8 & 21 & 22 & 10 & 25 & 41 & 42 & 17 \\
\hline 9 & 21 & 22 & 11 & 26 & 30 & 31 & 10 \\
\hline 10 & 21 & 22 & 17 & 27 & 30 & 31 & 11 \\
\hline 11 & 22 & 23 & 10 & 28 & 30 & 31 & 17 \\
\hline 12 & 22 & 23 & 11 & 29 & 31 & 32 & 10 \\
\hline 13 & 22 & 23 & 17 & 30 & 31 & 32 & 11 \\
\hline 14 & 26 & 27 & 10 & 31 & 31 & 32 & 17 \\
\hline 15 & 26 & 27 & 11 & 32 & 42 & 43 & 10 \\
\hline 16 & 26 & 27 & 17 & 33 & 42 & 43 & 11 \\
\hline 17 & 34 & 35 & 10 & 34 & 42 & 43 & 17 \\
\hline
\end{tabular}

TABLE 3: Individual loss reduction strategies (distribution transformer).

\begin{tabular}{|c|c|c|c|c|c|c|c|}
\hline $\begin{array}{l}\text { Strategy } \\
\text { number }\end{array}$ & $\begin{array}{l}\text { Head node of } \\
\text { branch }\end{array}$ & $\begin{array}{l}\text { Tail node of } \\
\text { branch }\end{array}$ & $\begin{array}{l}\text { New type } \\
\text { number }\end{array}$ & $\begin{array}{l}\text { Strategy } \\
\text { number }\end{array}$ & $\begin{array}{l}\text { Head node of } \\
\text { branch }\end{array}$ & $\begin{array}{l}\text { Tail node of } \\
\text { branch }\end{array}$ & $\begin{array}{l}\text { New type } \\
\text { number }\end{array}$ \\
\hline 1 & 18 & 19 & 11 & 5 & 51 & 53 & 12 \\
\hline 2 & 27 & 28 & 11 & 6 & 51 & 53 & 20 \\
\hline 3 & 51 & 52 & 12 & 7 & 55 & 56 & 12 \\
\hline 4 & 51 & 52 & 20 & 8 & 55 & 56 & 20 \\
\hline
\end{tabular}

TABLE 4: Individual loss reduction strategies (reactive power compensation).

\begin{tabular}{|c|c|c|c|c|c|}
\hline Strategy number & Load compensation node & Target power factor & Strategy number & Load compensation node & Target power factor \\
\hline 1 & 24 & 0.9 & 16 & 24 & 0.95 \\
\hline 2 & 19 & 0.9 & 17 & 19 & 0.95 \\
\hline 3 & 14 & 0.9 & 18 & 14 & 0.95 \\
\hline 4 & 24 & 0.91 & 19 & 24 & 0.96 \\
\hline 5 & 19 & 0.91 & 20 & 19 & 0.96 \\
\hline 6 & 14 & 0.91 & 21 & 14 & 0.96 \\
\hline 7 & 24 & 0.92 & 22 & 24 & 0.97 \\
\hline 8 & 19 & 0.92 & 23 & 19 & 0.97 \\
\hline
\end{tabular}


TABle 4: Continued.

\begin{tabular}{lccccc}
\hline Strategy number & Load compensation node & Target power factor & Strategy number & Load compensation node & Target power factor \\
\hline 9 & 14 & 0.92 & 24 & 14 & 0.97 \\
10 & 24 & 0.93 & 25 & 24 & 19 \\
11 & 19 & 0.93 & 26 & 14 & 0.98 \\
12 & 14 & 0.93 & 28 & 24 & 0.98 \\
13 & 24 & 0.94 & 29 & 19 & 0.98 \\
14 & 19 & 0.94 & 30 & 14 & 0.99 \\
15 & 14 & 0.94 & & 28 & \\
\hline
\end{tabular}

TABLE 5: Unit construction cost of related power equipment.

\begin{tabular}{lccc}
\hline Equipment & Type & Unit construction cost & Unit \\
\hline & YJV22-3*50 & 11.5 & $\times 10^{4} ¥ / \mathrm{km}$ \\
& YJV22-3*70 & 40 & $\times 10^{4} ¥ / \mathrm{km}$ \\
Line & YJV22-3*120 & 60 & $\times 10^{4} ¥ / \mathrm{km}$ \\
& JKLYJ-70 & 16 & $\times 10^{4} ¥ / \mathrm{km}$ \\
& JkLYJ-120 & 24 & $\times 10^{4} ¥ / \mathrm{km}$ \\
& S11-315 & 9 & $\times 10^{4} ¥$ \\
Transformer & S11-630 & 13 & $\times 10^{4} ¥$ \\
& S13-630 & 15 & $\times 10^{4} ¥$ \\
Reactive compensation & - & 80 & $¥ / \mathrm{kVar}$ \\
\hline
\end{tabular}

TABle 6: Cost-benefit calculation results of individual loss reduction strategies (distribution transformers).

\begin{tabular}{lcccc}
\hline Strategy number & Loss reduction rate $(\%)$ & Loss reduction benefit $\left(\times 10^{4} ¥\right)$ & Loss reduction cost $\left(\times 10^{4} ¥\right)$ & Cost-benefit ratio \\
\hline 1 & 0.028 & 144.2885 & 9 & 9 \\
2 & 0.006 & 144.2496 & 13 & 0.062 \\
3 & 0.006 & 144.2499 & 15 & 0.062 \\
4 & 0.006 & 144.2499 & 13 & 0.090 \\
5 & 0.020 & 144.2742 & 15 & 0.104 \\
6 & 0.020 & 144.2742 & 13 & 0.090 \\
7 & 0.006 & 144.2498 & 15 & 0.090 \\
8 & 0.006 & 144.2498 & 0.104 \\
\hline
\end{tabular}

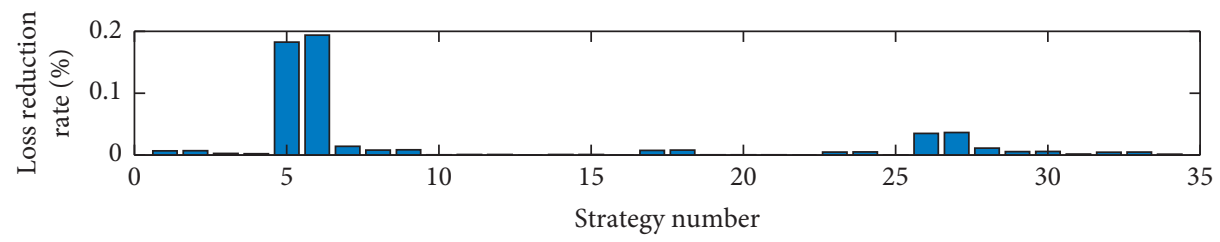

(a)

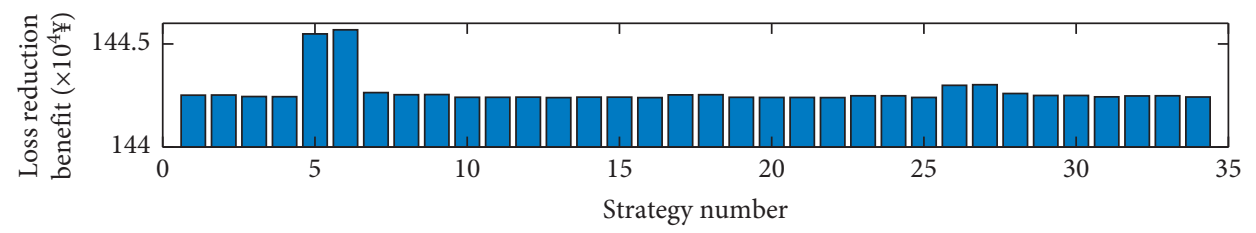

(b)

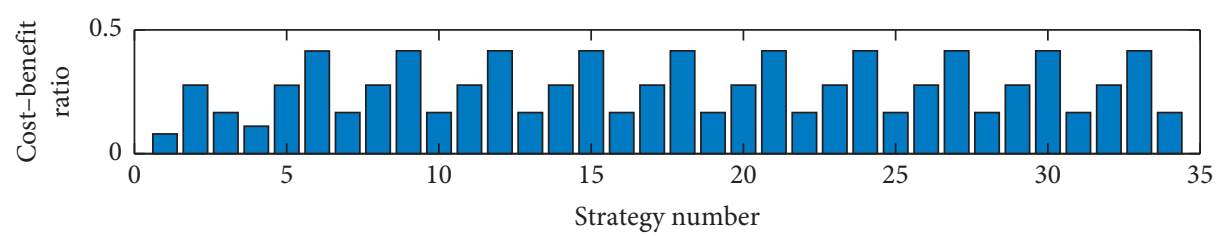

(c)

Figure 8: Cost-benefit calculation results of individual loss reduction strategies (distribution lines). 


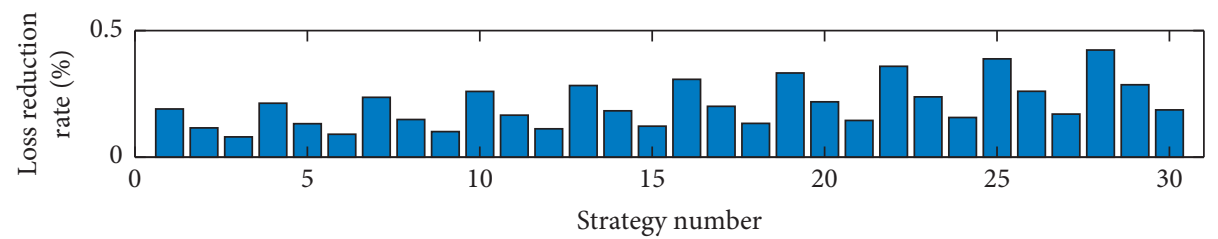

(a)

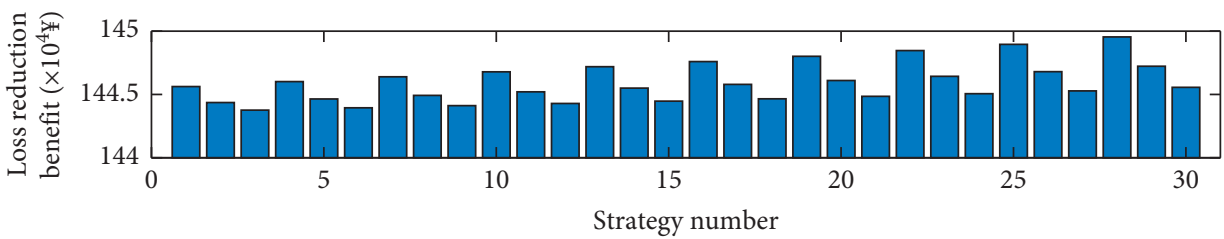

(b)

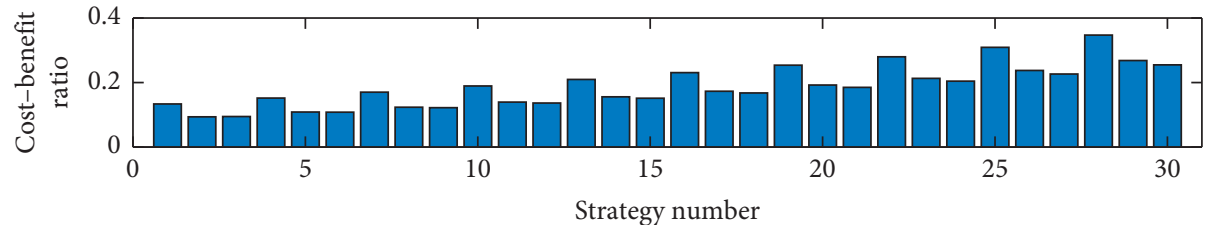

(c)

Figure 9: Cost-benefit calculation results of individual loss reduction strategies (reactive power compensation).

TABLE 7: Details of the individual optimal loss reduction strategies after sorting.

\begin{tabular}{lccccc}
\hline Number & Equipment & Corresponding strategy number & Number & Equipment & Corresponding strategy number \\
\hline 1 & Transformer & 1 & 11 & Line & 28 \\
2 & Transformer & 2 & 12 & Line & 31 \\
3 & Line & 1 & 13 & Line & 34 \\
4 & Transformer & 5 & 14 & Line & 10 \\
5 & Transformer & 3 & 15 & Line & 19 \\
6 & Transformer & 7 & 16 & Line & 25 \\
7 & Reactive power compensation & 2 & 17 & Line & 13 \\
8 & Reactive power compensation & 3 & 18 & Line & 16 \\
9 & Reactive power compensation & 1 & 19 & Line & 22 \\
10 & Line & 7 & & & \\
\hline
\end{tabular}

making its power factor reach the target value $0.9 \sim 0.99$.

\subsection{Cost-Benefit Analysis of Individual Loss Reduction} Strategy. Table 5 lists the unit construction cost of power equipment related to the loss reduction strategy selected in this paper.

From Tables 2 to 5, in the typical loss reduction scenario, the loss reduction rate, loss reduction benefit, and the corresponding cost-benefit ratio of each individual loss reduction strategy compared to the case where no loss reduction strategies can be calculated. The calculation results are shown in Table 6 and Figures 8 and 9.

3.5. Combined Loss Reduction Strategy Optimization. Based on the cost-benefit calculation results of each individual loss reduction strategy, the cost-benefit ratio order can be obtained by sorting the cost-benefit ratio from low to high, and the individual optimal loss reduction strategies for each loss reduction object can be determined. Table 7 and Figure 10 show the details of the individual optimal loss reduction strategies after sorting and the corresponding loss reduction rate, loss reduction benefit, and cost-benefit ratio, respectively.

The cost-benefit ratio of replacing the transformer type or reactive power compensation is lower. Thus, their comprehensive loss reduction benefit after considering the investment cost and the extent of loss reduction is better. This is because the distribution feeder has a relatively high cable rate. Most of the bare overhead conductors to be renovated are basically not on the main trunk. Therefore, when an individual loss reduction strategy is selected, the corresponding loss reduction rate is relatively small.

According to the sequence of the individual optimal loss reduction strategies listed in Table 7 , the alternative loss reduction strategy is combined in sequence starting from the first one. The power flow calculation is used to determine whether the target power loss rate value $6 \%$ is met. Figure 11 shows the result of the power loss rate after a combination of 


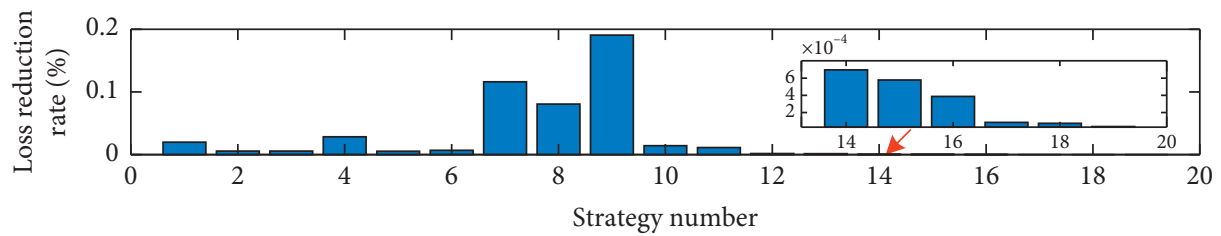

(a)

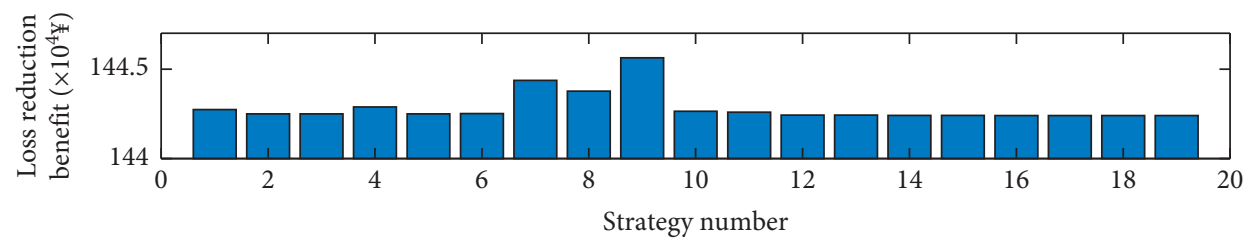

(b)

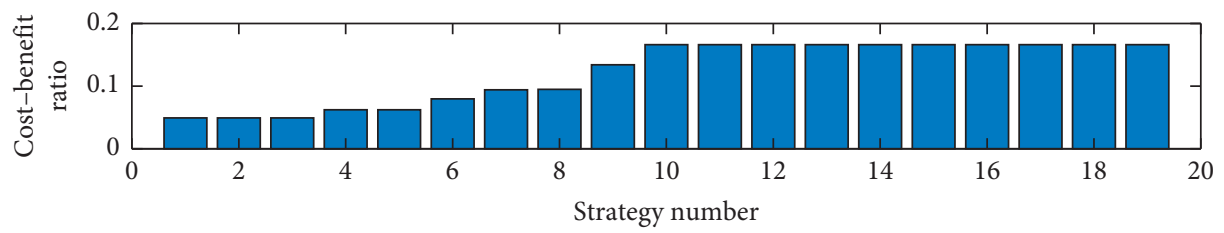

(c)

Figure 10: The cost-benefit calculation result of the individual optimal loss reduction strategy based on the cost-benefit ratio ranking.

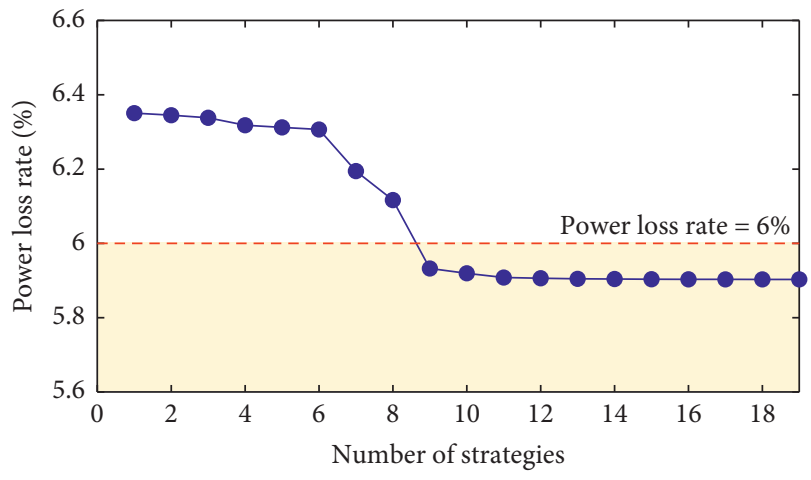

FIGURE 11: The result of the power loss rate after a combination of multiple strategies.

multiple strategies. It can be seen that when the top 9 kinds of loss reduction strategies are combined, the power loss rate of the feeder is already less than $6 \%$ at this time. After the loss reduction, the average power factor is 0.93 , the loss reduction benefit is 1.4499 million yuan, and the loss reduction cost is 1.152 million yuan.

\section{Conclusions}

In this paper, a framework of combined power loss reduction strategy optimization is proposed to improve the power loss reduction effect in the distribution network, containing three stages: weak point analysis of power loss, generation of loss reduction strategy, and combined loss reduction strategy optimization.

Experiments were conducted using the dataset from the Tianjin Electric Power Company in China. Based on the power flow calculation, the analysis result of power loss weak points was obtained. To achieve the purpose of targeted loss reduction, the corresponding power loss reduction strategies were generated considering three aspects of replacing distribution lines, distribution transformers, and reactive power compensation. The corresponding power loss reduction strategies were generated considering three aspects: replacing distribution lines, distribution transformers, and reactive power compensation. A combined power loss reduction strategy optimization model considering the comprehensive benefits of power loss reduction was established. In order to solve the problem that the enumeration methods were generally used in most of the existing research to solve the above model, which caused a low efficiency of power loss reduction, a method for solving the optimization model based on the cost-benefit ratio was proposed. The result of the case study suggested that the proposed loss reduction optimization method could effectively formulate a reasonable loss modification scheme in the distribution network.

\section{Data Availability}

The data used to support the findings of this study are included within the article.

\section{Conflicts of Interest}

The authors declare that there are no conflicts of interest regarding the publication of this paper.

\section{Acknowledgments}

This work was supported in part by National Natural Science Foundation of China under Grant 51807023 and Natural Science Foundation of Jiangsu Province under Grant BK20180382. 


\section{Supplementary Materials}

Table S1: line types. Table S2: transformer types. Table S3: branch information of the $10 \mathrm{kV}$ feeder in the actual distribution network. (Supplementary Materials)

\section{References}

[1] J. B. Leite and J. R. S. Mantovani, "Detecting and locating nontechnical losses in modern distribution networks," IEEE Transactions on Smart Grid, vol. 9, no. 2, pp. 1023-1032, 2018.

[2] J. Jun Wang, Z. Jiangping Yu, and J. Yu, "Practical calculation method of theoretical line loss in $10 \mathrm{kV}$ power distribution network based on Dscada," in Proceedings of the 2008 China International Conference on Electricity Distribution, Guangzhou, China, December 2008.

[3] C. M. P. Dos Santos, "Determination of electric power losses in distribution systems," in Proceedings of the 2006 IEEE/PES Transmission \& Distribution Conference and Exposition: Latin America, Caracas, Venezuela, August 2006.

[4] S. Zhang, X. Dong, Y. Xing, and Y. Wang, "Analysis of influencing factors of transmission line loss based on GBDT algorithm," in Proceedings of the 2019 International Conference on Communications, Information System and Computer Engineering (CISCE), Haikou, China, July 2019.

[5] J. F. Manirakiza and A. O. Ekwue, "Technical losses reduction strategies in a transmission network," in Proceedings of the 2019 IEEE Africon, Accra, Ghana, September 2019.

[6] M. Kundu, S. Jadhav, and K. Bagdia, "Technical loss reduction through active repair of distribution transformers: results from the field," in Proceedings of the 2017 7th International Conference on Power Systems (ICPS), Pune, India, December 2017.

[7] D.-S. He, W. Lin, and Z.-Q. Liang, "The Energy efficiency diagnosis research of regional power grid loss reduction," in Proceedings of the 2014 China International Conference on Electricity Distribution (CICED), Shenzhen, Chinadoi, September 2014.

[8] M. T. Au, T. M. Anthony, and M. Mohamad, "Strategies in technical loss reduction and it's impact on harmonic performance of distribution network," in in Proceedings of the 2009 IEEE Bucharest PowerTech, Bucharest, Romania, June 2009.

[9] L. Ying, M. Liu, L. Deng et al., "A comprehensive review of the loss reduction in distribution network," Power System Protection and Control, vol. 45, no. 19, pp. 162-169, 2017.

[10] D. K. Khatod, V. Pant, and J. Sharma, "Evolutionary programming based optimal placement of renewable distributed generators," IEEE Transactions on Power Systems, vol. 28, no. 2, pp. 683-695, 2013.

[11] B. R. Pereira, G. R. M. Martins da Costa, J. Contreras, and J. R. S. Mantovani, "Optimal distributed generation and reactive power allocation in electrical distribution systems," IEEE Transactions on Sustainable Energy, vol. 7, no. 3, pp. 975-984, 2016.

[12] L. Xie, Z. Tang, X. Huang et al., "Bi-layer dynamic reconfiguration of a distribution network considering the uncertainty of distributed generation and electric vehicles," Power System Protection and Control, vol. 48, no. 10, pp. 1-11, 2020.

[13] X. Wang, Z. Wei, G. Sun et al., "Multi-objective distribution network reconfiguration considering uncertainties of distributed generation and load," Electric Power Automation Equipment, vol. 36, no. 6, pp. 116-121, 2016.
[14] Y. J. Zhang, X. T. Zhang, Q. H. Li, L. Ran, and Z. X. Cai, "Gray theory based energy saving potential evaluation and planning for distribution networks," International Journal of Electrical Power \& Energy Systems, vol. 57, pp. 298-303, 2014.

[15] B. C. Neagu, O. Ivanov, and G. Georgescu, "Reactive power compensation in distribution networks using the bat algorithm," in Prceedings of the 2016 International Conference and Exposition on Electrical and Power Engineering (EPE), Iasi, Romania, October 2016.

[16] A. R. Gupta and A. Kumar, "Energy saving using D-STATCOM placement in radial distribution system under reconfigured network," Energy Procedia, vol. 90, pp. 124-136, 2016.

[17] S. A. Nowdeh, I. F. Davoudkhani, M. J. H. Moghaddam et al., "Fuzzy multi-objective placement of renewable energy sources in distribution system with objective of loss reduction and reliability improvement using a novel hybrid method," Applied Soft Computing, vol. 77, pp. 761-779, 2019.

[18] W. Huang, J. Jiang, W. Chen et al., "Study on differentiated energy saving and loss reduction countermeasures for medium-voltage and low-voltage distribution network," Power Capacitor \& Reactive Power Compensation, vol. 41, no. 5, pp. 0164-0170, 2020.

[19] L. Ding, "Research on the influence of aging and high resistance grounding fault on $10 \mathrm{kV}$ line," Jiangxi Electric Power, vol. 44, no. 8, pp. 35-38, 2020.

[20] J. A. H. A. Wong, "Algorithm as 136: a K-means clustering algorithm," Journal of the Royal Statistical Society, vol. 28, no. 1, pp. 100-108, 1979.

[21] M. Ester, H.-P. Kriegel, J. Sander, and X. Xu, “A density-based algorithm for discovering clusters in large spatial databases with noise," in Proceedings of the Second International Conference on Knowledge Discovery and Data Mining, Portland, OR, USA, August 1996. 